\title{
Einladung \\ zur \\ 68. Versammlung deutscher Naturforscher und Aerzte in Frankfurt a. M.
}

21. bis 26. September 1896.

Nachdem im vorigen Jahre in Lübeck einstimmig Frankfurt a. M. als Ort für die diesjährige Versammlung gewählt worden ist, haben die Unterzeichneten auf Aufforderung des Herrn Oberbürgermeisters und des Vorstandes der Gesellschaft das ehrenvolle Amt der Geschäftsführer gern und freudig übernommen. Schien doch die Wahl Frankfurts mit seiner glücklichen, centralen Lage an den Hanptverkehrsstrassen unseres deutschen Vaterlandes, mit seinen reichen geschichtlichen Erinnerungen und seiner an der Entwicklung der Wissenschaften den regsten Antbeil nehmenden Bürgerschaft eine sichere Gewähr für den würdigen Empfang und erfolgreichen Verlauf unserer Versammlung za bieten.

Das wohlwollende Entgegenkommen der städtischen und staatlichen Bebörden und die freudige Mitarbeit aller betheiligten Kreise Frankfurts haben uns während der bisherigen Vorbereitungen andauernd in der Hoffnung anf ein gutes Gelingen der Versammlung unterstützt, und mit Genugthunng weisen wir heute auf die ungewöhnlich grosse Anzahl von Vorträgen hin, die wir in dem nachstehenden Verzeichnisse der Abtheilungen zur Ankündigung bringen können.

So laden wir denn im Namen unserer hiesigen Fachgenossen und der gesammten Bürgerschaft Frankfurts alle deutschen Naturforscher und Aerzte und alle ausländischen Freunde der deutschen Forschung herzlichst ein, an der Versammlung theilzunehmen und mit ihr Einkehr zu halten in der alten Kaiserstadt am Main.

\section{Allgemeine Tagesordnung.}

Sonntag, den 20. September:

Morgens 10 Uhr: Sitzung des Vorstandes der Gesellschaft deutscher Naturforscher und Aerzte im grossen Conferenzzimmer des Hauptpersonenbahnhofs (Nordflügel).

Morgens 11 Uhr: Grundsteinlegung des Denkmals Samuel Thomas von Soemmerrings. Mittags 12 Uhr: Sitzung des wissenschaftlichen Ausschusses im grossen Conferenzzimmer des Hauptpersonenbahnhofs (Nordflügel).

Abends 8 Uhr: Begrüssung im Saalbau (mit Damen): Liedervorträge des Sängerchors des Frankfurter Lehrervereins.

\section{Montag, den 21. September:}

Morgens 9 Uhr: I. Allgemeine Sitzung im grossen Saale des Saalbaues.

1. Eröffnung durch den ersten Geschäftsfïhrer der Versammlung, Herrn Geh. San.-Rath Prof. Dr. med. Moritz Schmidt.

2. Begrüssungsansprachen.

3. Mittheilungen des Vorsitzenden der Gesellschaft deutscher Naturforscher und Aerzte, Herrn Geh.-Rath Professor Dr. med. Hugo v. Ziemssen (München). 
Einladung zur 68. Versamm]ung deutscher Naturforscher u. Aerzte in Frankfurt a. M.

4. Vortrag des Herrn Professor Dr. med. Hans Buchner (München): Biologie und Gesundheitslehre.

5. Vortrag des Herrn Geh. Hofrath Professor Dr. phil. Richard Lepsius (Darmstadt): Cultur und Eiszeit.

Nachmittags 3 Uhr: Bildung und Eröffnung der Abtheilungen. Wahl der Wablmänner für den wissenschaftlichen Ausschuss.

Abends 7 Uhr: Festvorstellung im Opernhause; Vorstellung im Schauspielhause. Nach denselben zwanglose gesellige Vereinigung.

\section{Dienstag, den 22. September:}

Morgens 9 Vhr: Sitzungen der Abtheilungen. Wahl der Wahlmänner für den wissenschaftlichen Ausschuss (sofern nicht am 21. September Nachmittags vollzogen).

Nachmittags: Sitzungen der Abtheilungen.

Abends 61/2 Chr: Festessen im Zoologischen Garten (mit Damen), nach demselben zwanglose gesellige Vereinigung daselbst.

\section{Mittroch, den 23. September:}

Morgens 9 Uhr: Wahl des wissenschaftlichen Ausschusses durch die Wahlmänner im grossen Saale des Saalbanes.

Abtheilungssitzungen, bezw. gemeinsame Sitzungen verschiedener Abtheilungen.

Gemeinsame Sitzung der Abtheilungen der medicinischen Hauptgruppe im grossen Saale des Saalbaues, Morgens 91/2 Uhr. Vorsitzender: Herr Geh. Medicinalrath Prof. Dr. med. Wilhelm His (Leipzig).

Zur Verhandlung kommen: Es haben Referate übernommen:

Herr Geh. Medicinalrath Prof. Dr. med. Paul Flechsig (Leipzig): Die Localisation der geistigen Vorgänge.

Herr Prof. Dr. med. L udwig Edinger (Frankfurt a. M.): Die Entwicklung der Gehirnbahnen in der Thierreihe.

Herr Geh.-Rath Prof. Dr. med. Ernst v. Bergmann (Berlin): Ueber Gehirngeschwülste.

Discussion. Die Uebertragung von Vorträgen, die für Abtheilungssitzungen angemeldet sind, auf diese gemeinsame Sitzung bleibt späterer Verständigung vorbehalten.

Nachmittags: Abtheilungssitzungen, bezw. gemeinsame Sitzungen verschiedener Abtheilungen.

Abends $81 / 2$ Uhr: Fest-Commers (mit Damen) in der Landwirthschaftlichen Halle, gegeben von der Stadt Frankfurt a. M.

\section{Donnerstag, den 24. September:}

Sitzungen der Abtheilungen.

Abends 8 Uhr: Festball im Palmengarten.

\section{Freitag, den 25. September:}

Morgens 9 Uhr: Geschäftssitzung der Gesellschaft im grossen Saale des Saalbaues. Morgens 91/2 Uhr: II. Allgemeine Sitzung daselbst.

1. Vortrag des Herrn Professor Dr. med. Max Verworn (Jena): Erregung und Lähmung.

2. Vortrag des Herrn Dr. med. Ernst Below (Berlin): Die praktischen Ziele der Tropenhygiene.

3. Vortrag des Herrn Geh. San.-Rath Professor Dr. med. Carl Weig ert Nachmittags: Ausflüge (Frankfurt a. M.): Neue Fragestellungen in der pathologischen Anatomie.

1. nach Darmstadt zum Besuche der Technischen Hochschule (Besichtigung der neuen Institute) und der. Landwirthschaftlichen Versuchsstation.

2. nach der Lungenheilanstalt Falkenstein, der Volksheilstätte Ruppertshain und Königstein i. T.

3. nach den Höchster Farbwerken zur Besichtigung der Serumabtheilung. 
Einladung zur 68. Versammlung deutscher Naturforscher u. Aerzte in Frankfurt a. M.

4. nach Bad Soden a. T.

5. nach Bad Nauheim.

Abends: 1. Zwanglose gesellige Vereinigung im Saalbau in Darmstadt.

2. Gesellige Vereinigung im Garten des Hôtel Pfaff in Königstein i. T.; Concert und Beleuchtung der Burgruine.

3. Gesellige Vereinigung auf der Kurhausterrasse in Bad Soden a. T., Concert und bengalische Beleuchtung des Kurparks.

4. Gesellige Vereinigung auf der Karhausterrasse in Bad Nauheim, Concert und Beleuchtung des grossen Sprudels.

5. Festconcert der Museumsgesellschaft im grossen Saale des Saalbaues in Frankfurt a. M.

\section{Sonnabend, den 26. September:}

1. Tagesausflug nach Homburg v. d. H. Besichtigung des Quellengebietes, Badehauses und Saalburgmuseums. Gemeinsames Frühstück auf der Terrasse, gegeben von der Stadt Homburg. Fahrt nach der Saalburg, Besichtigung derselben unter fachmännischer Führung. Rückkehr nach Homburg. Mahl im Kurhause, Gartenfest und Brillantfeuerwerk im Kurpark.

2. Tagesausflug nach Marburg i. H. Führung zur Besichtigung der Institute der Universität und der Sehenswürdigkeiten der Stadt. Musikfrühschoppen auf Bopp-Lederer's Terrasse, gegeben von der Stadt Marburg. Gemeinschaftliches Mittagsessen im Local der Museumsgesellschaft, Garten- oder Waldfest. Abends eventuell Beleuchtung des Schlosses.

3. Vormittagsausflug nach Giessen (eventuell mit dem Ausfluge nach Marburg zu verbinden). Besichtigung der medicinischen Universitätsinstitute, sodann Frühstück in der Actienbrauerei, gegeben von der Stadt Giessen.

\section{Erläuterungen zur Tagesordnung.}

Anmeldungen zur Mitgliedschaft erfolgen schriftlich beim Schatzmeister der Gesellschaft, Herrn Dr. Carl Lampe - Vischer, Leipzig, an der I. Bürgerschule 2, vom 20. September an auch persönlich in der Geschäftsstelle der 68. Versammlung, in der Turnhalle des städtischen Gymnasiums zu Frankfurt a. M., Junghofstrasse 16 (\$1 der Geschäftsordnung).

Theilnehmer an der Versammlung kann jeder werden, welcher sich für Naturwissenschaften und Medicin interessirt.

Die Theilnehmerkarte ist von jetzt ab gegen Einsendung $\nabla$ on 15 Mark an den Cassirer der Geschäftsführung der 68. Versammlung deutscher Naturforscher und Aerzte, Herrn Hugo Metzler in Frankfurt a. M., am Salzhaus 3, zu erhalten. Sie berechtigt zum Bezug des Festabzeichens, des Tageblattes, der Festgabe und sonstiger für die Theilnehmer bestimmter Drucksachen, sowie zur Theilnahme an den Sitzungen und Festlichkeiten, und ferner zur Entnahme von Damenkarten zum Preise von 6 Mark.

Die später erscheinenden ,Verhandlungen" werden den Mitgliedern der Gesellschaft dentscher Naturforscher und Aerzte, soweit sie auf dieselben abonnirt oder an der Versammlung Theil genommen haben, zugestellt. Für Mitglieder, welche den Betrag für Bezug der Verhandlungen bereits an den Schatzmeister eingezahlt haben, ermässigt sich der Preis der Theilnehmerkarte um 6 Mark. Bei Lösung derselben ist die Quittung des Schatzmeisters vorzulegen. Theilnehmer, welche nicht Mitglieder der Gesellschaft sind, erhalten die "Verhandlungen" zum Preise von 6 Mark, wenn sie sich in eine in der Geschäftsstelle aufliegende Liste einzeichnen. 
Einladung zur 68. Versammlung deutscher Naturforscher u. Aerzte in Frankfurt a. M.

Ein besonderer Damenausschuss hat es sich zur Aufgabe gemacht, für die Unterhaltung der Damen Sorge zu tragen, insbesondere ihnen die Sehenswürdigkeiten und Wohlthätigkeits-Anstalten Frankfurts zugänglich zu machen. Er beabsichtigt an einem Nachmittage der Versammlungswoche bei günstiger Witterung die Veranstaltung eines Gartenfestes.

Die Damen erhalten ebenfalls das Festabzeichen, nehmen an den allgemeinen Sitzungen, an allen, insbesondere an den rom Damenausschusse für sie veranstalteten Festlichkeiten Theil und empfangen die für sie be. stimmte Festgabe.

Gegen Vorzeigen der Theilnehmer- bezw. Damenkarte und Vermerk anf derselben werden für einzelne Veranstaltungen besondere Karten ausgegeben.

Vorausbesteliungen von Wohnungen in Hôtels, sowie in Privathäusern nimmt das Bïreau des Wohnungsausschusses, Stiftstrasse 30 , von jetzt ab entgegen. Man wolle sich der beiliegenden Karte bedienen und Anmeldungen thunlichst beschleunigen, da infolge des in Frankfurt erfahrungsgemäss sehr starken Fremdenverkehrs im September die angemessene Unterbringung unserer Gäste nicht ganz leicht sein dürfte. Auch in Homburg . d. H. und Soden i. T. ist dem Wohnungsausschusse eine grössere Anzahl von Wohnungen für die Theilnehmer an der Versammlung theils unentgeltlich, die meisten gegen Entgelt (2 Mark einschl. Licht, Bedienung und erstes Friuhstïck), zur Verfïgung gestellt worden. Mit beiden Orten besteht häufige Eisenbahnverbindung, so dass dort wohnende Theilnehmer bequem Morgens nach Frankfurt kommen und Abends zurückfahren können.

Jedenfalls kann der Wohnungsausschuss keine Gewähr dafür übernehmen, dass den nach dem 10. September eingehenden Wünschen noch wird Rechnung getragen werden können.

Es besteht aber bei den Einwohnern Frankfurt's der lebhafte Wunsch, Naturforscher und Aerzte in ihren Häusern gastlich aufzunehmen, und bitten wir unter den dargelegten Verhältnissen von dieser Einladung recht reichlich Gebrauch machen zu wollen.

Im Hauptpersonenbahnhofe wird von Sonnabend den 19. bis Mittwoch den 23. September ein Empfangs- und Auskunftsbureau während des ganzen Tages geöffnet sein. Ebendaselbst wird in dieser Zeit anch der Nachweis von Wohnungen ertheilt und die Besorgung von Handgepäck and grösseren Gepäckstiicken vom Bahnhofe in die betr. Quartiere und die Aufbewahrung von Handgepäck übernommen werden.

Die Geschäftsstelle in der Turnhalle des städtischen Gymnasiums, Junghofstrasse 16, wird für die Dauer der Versammlung zur Einzeichnung in die Präsenzliste, zur Ausgabe der Festkarten, des Tageblattes n. s. w. am Sonnabend, den 19. September von 4-8 Uhr Nachmittags, am Sonntag, den 20. von Morgens 7 Uhr bis 12 Uhr Nachts, am Montag, den 21. von $8 \mathrm{Uhr}$ Morgens bis $8 \mathrm{Uhr}$ Abends und vom 22. bis 25. September von 8-12 Uhr Vormittags und von 3-6 Uhr Nachmittags geöffnet sein.

Frankfurt a. M., im Juli 1896.

Prof. Moritz Schmidt,

Geheimer Sanitätsrath,

I. Gescbäftsführer.
Prof. Walter König,

Docent am Physikalischen Verein,

II. Geschäftsführer. 